\title{
Thermal Instability and Alfvén Waves in the Formation of Quasar Clouds
}

\author{
D. R. Gonçalves, V. Jatenco-Pereira, and R. Opher \\ Instituto Astronômico e Geofísico, Universidade de São Paulo \\ Av. Miguel Stéfano 4200, 04301-904 São Paulo - SP, Brazil
}

\begin{abstract}
We use thermal instability as a condensation mechanism to form the characteristic clouds of the BLR of quasars. We include in the total heat-loss function the influence of the heating caused by the damping of Alfvén waves. We study the damping mechanisms: nonlinear, resonance surface, and turbulent. From this analysis, we show that this heating mechanism plays an important role in the formation of these clouds.
\end{abstract}

\section{Introduction}

In general, astronomical objects are formed by self-gravitation. However, some objects cannot be explained by this process. If the thermal equilibrium of the medium is a balance between energy gains and radiative losses, instability results if, near equilibrium, the losses increase with decreasing temperature. Then a cooler than average region cools more effectively than its surroundings, and its temperature drops rapidly below the initial equilibrium value (Parker 1953, Field 1965).

In general, it is believed that the material that is emitting lines in the broad-line region of active galactic nuclei (AGNs) is distributed in small clouds (Osterbrock \& Mathews 1986). Krolik et al. (1981) argued that an intercloud medium could be in pressure equilibrium with the clouds and two states of radiative equilibrium should exist, one at $T \approx 10^{8} \mathrm{~K}$ and the other at $T \approx 10^{4} \mathrm{~K}$.

We know that there is certainly ionized gas in AGNs, and there is almost certainly rotation. Under these circumstances, a magnetic field may arise naturally. We know also that the central regions of AGNs are highly perturbed due to the high variability of the central source. Perturbations of the magnetic field can create Alfvén waves.

In the literature one can find works which consider the thermal instability condensation mode in the formation of BLR clouds of quasars (Krolik 1988, Mathews \& Doane 1990, Gonçalves et al. 1993). Here we consider the possibility that the broad-line region (BLR) clouds of quasars are formed via thermal instability in the presence of Alfvén waves. We study the heating mechanisms for Alfvén waves (nonlinear damping, resonance-surface damping, and turbulent damping) in the formation of a two-phase medium for broad-line clouds of quasars. 


\section{The Model}

Consider a spherical collapsing cloud. For the collapsing cloud, the crosssectional area perpendicular to a magnetic field, $A$, is $\propto \rho^{-2 / 3}$ and $B \propto A^{-1} \propto$ $\rho^{2 / 3}$, where $\rho$ is the mass density of the gas and $B$ is the magnetic field. The damping length in each case is $L=v_{A} / \Gamma$ (i.e., the ratio between the Alfvén velocity and the damping rate). Using a characteristic density of the BLR clouds, $n \approx 10^{10} \mathrm{~cm}^{-3}$, we evaluate the Alfvénic heating for each damping mechanism. The complete function of heating and cooling, $H(T, \rho)$, includes recombination, thermal bremsstrahlung, Compton and inverse-Compton effects, a fit to the radiative losses via emission-line cooling, obtained using Ferland's photoionization code CLOUDY (Ferland 1993), and heating due to the damping of Alfvén waves.

\section{Results}

We find that thermal instability can occur at the pressures inferred for BLR clouds, $\sim 10^{-2} \mathrm{dyn} \mathrm{cm}^{-2}$; in particular, we find that the broad-line emitting clouds of quasars $\left(T_{c} \approx 1.4-3.0 \times 10^{4} \mathrm{~K}\right.$ and $\left.n_{c} \approx 10^{9} \mathrm{~cm}^{-3}\right)$ can be kept in thermal equilibrium with the hot intercloud medium $\left(T_{0} \approx 10^{7} \mathrm{~K}\right.$ and $n_{0} \approx$ $\left.10^{6} \mathrm{~cm}^{-3}\right)$. As we work with three distinct Alfvénic heating mechanisms, we performed calculations with each one and compared their efficiency. It is evident that the expected cloud temperature depends on the heating and cooling processes assumed in the thermal equilibrium. We find that turbulent Alfvénic heating reaches lower temperatures than those related to nonlinear and resonance surface Alfvénic heating. We show that quasar clouds can be formed at the observed pressure by a thermal instability in the presence of Alfvén wave heating.

Acknowledgments. D. R. G. thanks the Brazilian agency FAPESP for support, and V.J.P. and R. O. would like to thank the Brazilian agency CNPq for partial support.

\section{References}

Ferland, G. J. 1993, in HAZY: A Brief Introduction to CLOUDY 84.00, (Columbus: OSU Astronomy Department Internal Report).

Field, G. B. 1965, ApJ, 142, 531,

Gonçalves, D. R., Jatenco-Pereira, V., \& Opher R. 1993, ApJ, 414, 57.

Krolik, J. H., McKee, C.F., \& Tarter, C. B. 1981, ApJ, 249, 422.

Krolik, J. H. 1988, ApJ, 325, 148.

Mathews, W. G., \& Doane, J.S. 1990, ApJ, 352, 423.

Osterbrock, D.E., \& Mathews, W.G. 1986, ARA\&A, 24, 171.

Parker, E. N. 1953, ApJ, 117, 431. 